Punjab University Journal of Mathematics (2021),53(12),881-892

https://doi.org/10.52280/pujm.2021.531204

\title{
Development of the structure of q-Rung Orthopair Fuzzy Hypersoft Set with basic Operations
}

\author{
Salma Khan, Muhammad Gulistan and Hafiz Abdul Wahab \\ Department of Mathematics and Statistics, Hazara University, Mansehra, Pakistan Email: \\ salmakhan359@gmail.com*, gulistanmath@hu.edu.pk, wahabmaths@yahoo.com
}

Received: 24 August 2021 / Accepted: 01 December 2021, / Published online: 24 Decem-ber, 2021

\begin{abstract}
This article introduces a new concept, called q-rung orthopair fuzzy hypersoft set (q-ROF Hypersoft sets). We introduce a theme of some fundamental operations such as q-ROF Hypersoft-subset, q-ROF Hypersoft null-set, q-ROF Hypersoft absolute-set, union, intersection, and complement. We also present "AND" and "OR" operators. To check out the validity of q-ROF Hypersoft sets, we use some numerical examples.
\end{abstract}

Key Words: Soft Set, Hypersoft Set, Fuzzy Hypersoft Set, q-Rung Orthopair Fuzzy Hypersoft Set.

\section{INTRODUCTION}

In all branches of science, some problems are full of uncertainties and impacts. Some of these problems are related to human life, and classical methods cannot solve such situations because they cannot handle the various ambiguities involved. The purpose of Zadeh's fuzzy set theory was to represent facts and notions that were imprecise and had several meanings. Therefore, Zadeh [23] introduced fuzzy sets as an extension of classical set theory. After Zadeh, many researchers $[19,3,5,20]$ worked on fuzzy sets and defined different fuzzy operations, fuzzy relations, fuzzy functions, fuzzy measures, fuzzy models, and formal structures, etc. The soft set supposition is the induction of a fuzzy set supposition presented by Molodtsov [9] to trade unpredictably in a parametric model. He identified strains and troubles in mathematical representations and addressed the complications by proposing a soft set theory. After Molodtsov, many researchers such as Saeed et al. [12] used the soft set and introduced soft elements and soft members in the soft set. Gong et al. [6] introduced the concept of a bijective soft set and investigated its properties. Cayman et al. [4] extended the soft theory to fuzzy soft set theory by giving membership function to each element of the mapping of soft function. They also developed aggregation operators of fuzzy soft sets and gave an application in real-life problems. Maji $[11,8]$ expanded the soft set scheme into a fuzzy soft set and neutrosophic soft set theory. Smarandache [16] developed the concept of soft-set to Hypersoft sets to deal with vagueness, inconsistency, and ambiguity in decision-making problems. After that, Saeed et al. [13, 14, 15] presented the basics of Hypersoft sets, aggregation operators, and characterized the fundamental axioms, properties, and laws of a hypersoft set, and discussed decision-making techniques. In 
addition, Yolcu et al. and Zulqarnain [22, 21, 10, 24] has also worked on fuzzy hypersoft sets, intuitionistic Fuzzy hypersoft sets and neutrosophic hypersoft topological spaces, and Pythagorean fuzzy hypersoft set to process uncertain data parametrically. As in the above work, the linear inequalities between membership grade and non-membership grade are examined. However, if the decision-maker goes steady with object membership degree $=.9$ and non-membership degree $=.8$, then $.9+.8 \not \leq 1$. Here, we can see that theory like the intuitionistic fuzzy set failed, introduced by Atanassov [2]. After that, Yager [18] defined a new structure known as the Pythagorean fuzzy set. In the Pythagorean fuzzy set, we check that if we increase the power to 2 , then $.9^{2}+.8^{2} \nless 1$, we can see that the Pythagorean fuzzy set theory has also failed. Yager and Ali $[17,1]$ also introduced a new q-rung orthopair fuzzy set theme. In which the degree of membership $u^{q}$ and non-membership $v^{q}$ satisfy $u^{q}+v^{q} \leq 1$ and $q \geq 1$, and Liu et al. [7] defined aggregation operator for decision making problem in q-rung orthopair fuzzy environment. Therefore, q-ROF-sets have more ability to process ambiguous data than IFS and PFS because the q-ROF sets generalize the intuitionistic fuzzy set and the Pythagorean fuzzy set. The q-ROF set is more flexible and effective because it handles more complex data than IFS and PFS.

The main objective of this study is defined as follows:

- We develop a structure, namely q-ROF Hypersoft sets.

- We also establish the essential operation of our proposed design, i.e., subset, null set, union, intersection, and complement.

- We formulate examples for our proposed structure .

Section 2 presents some basic concepts related to fuzzy sets and soft sets. In section 3, we develop a new structure known as q-ROF Hypersoft sets. Further, the essential operation of q-ROF Hypersoft sets, with an example, is also defined in section 3, and section 4 presents a rigorous study, and section 5 presents limitations. In the end, section 6 offers the conclusion and future work.

\section{NotATIONS AND PRELIMINARIES}

This section will recall some fundamental concepts such as soft set, fuzzy soft set, hypersoft set, and Pythagorean fuzzy hypersoft set that will help us establish the framework for the following article.

Definition 2.1. [9] Let $Y$ be a universal set, $\Gamma$ be an attributive set with respect to universal set $Y$, and $\tau \subseteq \Gamma$. Then a pair $(K, \tau)$ is called a soft set over $Y$, where $K$ is a mapping such that

$$
K: \tau \rightarrow P^{Y}
$$

where $P^{Y}$ represents the collection of all subsets of $Y$. A pair $(K, \tau)$ can be expressed as

$$
(K, \tau)=\left\{\langle e, K(e)\rangle \mid e \in \tau, K(e) \in P^{Y}\right\}
$$

Definition 2.2. [4] Let $Y$ be a set of discourse, $\Gamma$ be an attributive set with respect to universal set $Y$, and $\tau \subseteq \Gamma$. Then, a pair $(K, \tau)$ is called a fuzzy soft set where $K$ is a mapping such that

$$
K: \tau \rightarrow F^{Y}
$$


where $F^{Y}$ represents the collection of all fuzzy subsets of $Y$. A pair $(K, \tau)$ can be expressed as

$$
(K, \tau)=\left\{\langle e, K(e)\rangle \mid e \in \tau, K(e) \in F^{Y}\right\}
$$

where

$$
K(e)=\left\{\left\langle y, \mu_{F}(\ddot{y})\right\rangle \mid \ddot{y} \in Y\right\}
$$

and

$$
\mu_{F}(\ddot{y}) \in[0,1]
$$

Definition 2.3. [16] Let $Y$ be a set of discourse and $\tau=\left\{a_{1}, a_{2}, a_{3}, \ldots, a_{n}\right\}$ be a set of attributes, whose attributive values belong to the sets $\tau_{1}, \tau_{2}, \ldots, \tau_{n}$ respectively, with condition $\tau_{i} \cap \tau_{j}=\phi$, for $i \neq j$ and $i, j \in\{1,2, \ldots, n\}$. A pair $(\Re, \bar{A})$ is called Hypersoft set over the universal set $Y$, where $\bar{A}=\tau_{1} \times \tau_{2} \times \ldots \times \tau_{n}$ and where $\Re$ is the mapping such that

$$
\Re: \bar{A} \rightarrow P(Y)
$$

where $P(Y)$ represents the collection of all subsets of $Y$. A pair $(\Re, \bar{A})$ can be expressed as

$$
(\Re, \bar{A})=\{\langle\bar{a}, \Re(\bar{a})\rangle \mid \bar{a} \in \bar{A}, \Re(\bar{a}) \in P(Y)\}
$$

Definition 2.4. [2] Let $Y$ be a set of discourse and $\tau=\left\{a_{1}, a_{2}, a_{3}, \ldots, a_{n}\right\}$ be a set of attributes, whose attributive values belong to the set $\tau_{1}, \tau_{2}, \ldots, \tau_{n}$ respectively, with condition $\tau_{i} \cap \tau_{j}=\phi$, for $i \neq j$ and $i, j \in\{1,2, \ldots, n\}$. Assume a relation $\tau_{1} \times \tau_{2} \times \ldots \times \tau_{n}=$ $\bar{A}=\left\{k_{1 h}, k_{1 m}, \ldots, k_{1 n}\right\}$ be a family of sub-attributes, where $1 \leq h \leq \alpha, 1 \leq m \leq \beta$ and $1 \leq n \leq \gamma$ and $\alpha, \beta$ and $\gamma \in N$. Then a pair $(\Re, \bar{A})$ is called Pythagorean fuzzy hypersoft set over the universal set $Y$ and $\Re$ is a mapping

$$
\begin{aligned}
& \Re: \tau_{1} \times \tau_{2} \times \ldots \times \tau_{n} \rightarrow \operatorname{PFHS}^{(Y)} \\
& \Re: \bar{A} \rightarrow P F H S^{(Y)}
\end{aligned}
$$

,which is defined as $\Re\left(\tau_{1} \times \tau_{2} \times \ldots \times \tau_{n}\right)=\left\{\left(\bar{a}, \Re_{\bar{A}}(\bar{a})\right): \bar{a} \in \bar{A}, \Re_{\bar{A}}(\bar{a}) \in \operatorname{PFH}^{Y} \in[0,1]\right\}$, where

$$
\Re_{\bar{A}}(\bar{a})=\left\{\left\langle\ddot{y}, \psi_{\bar{A}(\bar{a})}(\ddot{y}), \tilde{\psi}_{\bar{A}(\bar{a})}(\ddot{y})\right\rangle \mid \ddot{y} \in Y\right\}
$$

where PFHS $S^{(Y)}$ represents the collection of all PF subsets of Y. Here $\psi$ and $\tilde{\psi}$ denotes the Mem and NMem grades of an object $\ddot{y} \in Y$ to a pair $(\Re, \bar{A})$, respectively, and hold the restriction that $0 \leq\left(\psi_{\bar{A}(\bar{a})}(\ddot{y})\right)^{2}+\left(\tilde{\psi}_{\bar{A}(\bar{a})}(\ddot{y})\right)^{2} \leq 1$. For sake of simplicity we write $\bar{A}$ for $\tau_{1} \times \tau_{2}, \ldots, \tau_{n}$ and $\bar{a}$ for an element of the set $\bar{A}$. Where $(\Re, \bar{A})=(\psi, \tilde{\psi})$ represents $a$ PFHS number.

\section{Q-Rung OrthoApir FuZzy Hypersoft SET}

In this section, we introduce q-rung orthopair fuzzy hypersoft set (q-ROF Hypersoft sets) and some basic operations like union, intersection, complement, and "OR," "AND" operators with numerical examples. 
Definition 3.1. Let $Y$ be the universal set and $a_{1}, a_{3}, a_{3}, \ldots, a_{n}$ be $n$ distinct attributes/parameters concerning $Y$, whose corresponding attributive values belong to the sets $\tau_{1}, \tau_{2}, \ldots, \tau_{n}$ respectively, such that $\tau_{i} \cap \tau_{j}=\phi$, for $i \neq j$. Suppose a relation $\tau_{1} \times \tau_{2} \times \ldots \times \tau_{n}=$ $\bar{A}=\left\{k_{1 h}, k_{1 m}, \ldots, k_{1 n}\right\}$ be a family of sub-parameters, where $1 \leq h \leq \alpha, 1 \leq m \leq \beta$ and $1 \leq n \leq \gamma$ and $\alpha, \beta$ and $\gamma \in N$. A pair $(\Re, \bar{A})$ is called q-rung orthopair fuzzy hypersoft set over the universal set $Y$, where $\Re$ is a function given as $\Re: \bar{A} \rightarrow q-$ $R O F S^{(Y)}$. It is a family of $q-R O F$ subsets of $Y$, and it can be defined as $(\Re, \bar{A})$ $=\left\{\left(\bar{a}, \Re_{\bar{A}}(\bar{a})\right): \bar{a} \in \bar{A}, \Re_{\bar{A}}(\bar{a}) \in q-\operatorname{ROF} S^{Y} \in[0,1]\right\}$,

where $\Re_{\bar{A}}(\bar{a})=\left\{\left\langle\ddot{y}, \psi_{\bar{A}(\bar{a})}(\ddot{y}), \tilde{\psi}_{\bar{A}(\bar{a})}(\ddot{y})\right\rangle \mid \ddot{y} \in Y\right.$ and $\left.q \geq 1\right\}$. Here $\psi$ and $\tilde{\psi}$ denotes the Mem and NMem grades of an object $\ddot{y} \in Y$ to a pair $(\Re, \bar{A})$ respectively, and hold the restriction that $0 \leq\left(\psi_{\bar{A}(\bar{a})}(\ddot{y})\right)^{q}+\left(\tilde{\psi}_{\bar{A}(\bar{a})}(\ddot{y})\right)^{q} \leq 1$ and $q \geq 1$. For sake of simplicity we write $\bar{A}$ for $\tau_{1} \times \tau_{2} \times \ldots \times \tau_{n}$ and $\bar{a}$ for an element of the set $\bar{A}$. Where $q-R O F H S N$ can be express as $(\Re, \bar{A})=\left(\psi_{\bar{A}\left(\bar{a}_{i j}\right)}, \tilde{\psi}_{\bar{A}\left(\bar{a}_{i j}\right)}\right)$.

Example 1 Let $\mathrm{Y}$ be the set of decision makers to decide best laptop given as $Y=$ $\left\{y_{1}, y_{2}, y_{3}, y_{4}\right\}$ also consider the set of attributes as

- $\tau_{1}$ represents Laptop RAM

- $\tau_{2}$ represents Laptop type

- $\tau_{3}$ represents laptop hard drives

Then their corresponding attributive sets can be

$\tau_{1}=\left\{\mathrm{a}_{11}=8 \mathrm{~GB}, \mathrm{a}_{12}=16 \mathrm{~GB}, \mathrm{a}_{13}=32 \mathrm{~GB}\right\}$

$\tau_{2}=\left\{\mathrm{a}_{21}=\mathrm{HP}, \mathrm{a}_{22}=\right.$ Dell $\}$

$\tau_{3}=\left\{\right.$ a $_{31}=$ SATA, , $\left._{32}=\mathrm{SSD}\right\}$

Suppose $\mathrm{A}_{1}=\left\{\underset{\mathrm{s}}{\mathrm{a}_{11}}, \mathrm{a}_{12}\right\}, \mathrm{A}_{2}=\left\{\mathrm{a}_{21}\right\}, \mathrm{A}_{3}=\left\{\underset{\mathrm{s}}{\mathrm{a}_{31}}, \mathrm{a}_{32}\right\}$, and $\mathrm{B}_{1}=\left\{\mathrm{a}_{11}\right\}, B_{2}=$ $\left\{\mathrm{a}_{21}, \mathrm{a}_{22}\right\}, B_{3}=\left\{\mathrm{a}_{31}, \mathrm{a}_{32}\right\}$ are subsets of $\tau_{i}$ for $\mathrm{i}=1,2,3$.

Then $\bar{A}=\tau_{1} \times \tau_{2} \times \tau_{3}$ will have 3 -tuple elements and we suppose $\mathrm{q}=5$,

$$
\bar{A}=\left\{\bar{a}_{1}, \bar{a}_{2}, \bar{a}_{3}, \bar{a}_{4}, \bar{a}_{5}, \bar{a}_{6}, \bar{a}_{7}, \bar{a}_{8}, \bar{a}_{9}, \bar{a}_{10}, \bar{a}_{11}, \bar{a}_{12}\right\}
$$

$$
\begin{aligned}
& \bar{a}_{1}=\left(\mathrm{a}_{11}, \mathrm{a}_{21}, \mathrm{a}_{31}\right), \bar{a}_{2}=\left(\mathrm{a}_{11}, \mathrm{a}_{21}, \mathrm{a}_{32}\right), \bar{a}_{3}=\left(\mathrm{a}_{11}, \mathrm{a}_{22}, \mathrm{a}_{31}\right), \bar{a}_{4}=\left(\mathrm{a}_{11}, \mathrm{a}_{22}, \mathrm{a}_{32}\right), \\
& \bar{a}_{5}=\left(\mathrm{a}_{12}, \mathrm{a}_{21}, \mathrm{a}_{31}\right), \bar{a}_{6}=\left(\mathrm{a}_{12}, \mathrm{a}_{21}, \mathrm{a}_{32}\right), \bar{a}_{7}=\left(\mathrm{a}_{12}, \mathrm{a}_{22}, \mathrm{a}_{31}\right), \bar{a}_{8}=\left(\mathrm{a}_{12}, \mathrm{a}_{22}, \mathrm{a}_{32}\right), \\
& \bar{a}_{9}=\left(\mathrm{a}_{13}, \mathrm{a}_{21}, \mathrm{a}_{31}\right), \bar{a}_{10}=\left(\mathrm{a}_{13}, \mathrm{a}_{21}, \mathrm{a}_{32}\right), \bar{a}_{11}=\left(\mathrm{a}_{13}, \mathrm{a}_{22}, \mathrm{a}_{31}\right), \bar{a}_{12}=\left(\mathrm{a}_{13}, \mathrm{a}_{22}, \mathrm{a}_{32}\right)
\end{aligned}
$$

suppose

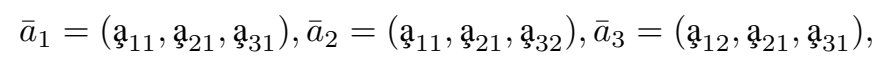

$$
\begin{aligned}
& \bar{a}_{4}=\left(\mathrm{a}_{12}, \mathrm{a}_{21}, \mathrm{a}_{32}\right)
\end{aligned}
$$

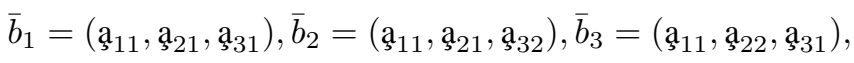

$$
\begin{aligned}
& \bar{b}_{4}=\left(\underset{3}{a_{11}}, \underset{3}{a_{22}}, \underset{3}{\mathrm{a}} 32\right)
\end{aligned}
$$


Then two q-ROF Hypersoft sets $(\Re, \bar{A})$ and $(\mathrm{G}, \bar{\beta})$ can be written as

$$
\begin{array}{r}
(\Re, \bar{A})=\left\{\begin{array}{l}
\left\langle\bar{a}_{1},\left\{\left(y_{1},(.6, .8)\right),\left(y_{2},(.7, .9)\right),\left(y_{3},(.8, .6)\right),\left(y_{4},(.5, .9)\right)\right\}\right\rangle, \\
\left\langle\bar{a}_{2},\left\{\left(y_{1},(.5, .8)\right),\left(y_{2},(.7, .6)\right),\left(y_{3},(.9, .5)\right),\left(y_{4},(.9, .8)\right)\right\}\right\rangle, \\
\left.\left\langle\bar{a}_{3},\left\{\left(y_{1},(.6, .8)\right),\left(y_{2},(.7, .6)\right),\left(y_{3}, .7, .9\right)\right),\left(y_{4},(.6, .9)\right)\right\}\right\rangle, \\
\left\langle\bar{a}_{4},\left\{\left(y_{1},(.9, .7)\right),\left(y_{2},(.9, .7)\right),\left(y_{3},(.7, .8)\right),\left(y_{4},(.8, .9)\right)\right\}\right\rangle,
\end{array}\right\} \\
(G, \bar{\beta})=\left\{\begin{array}{l}
\left\langle\bar{b}_{1},\left\{\left(y_{1},(.9, .7)\right),\left(y_{2},(.8, .9)\right),\left(y_{3},(.7, .9)\right),\left(y_{4},(.8, .7)\right)\right\}\right\rangle, \\
\left.\bar{b}_{2},\left\{\left(y_{1},(.7, .8)\right),\left(y_{2},(.7, .8)\right),\left(y_{3},(.9, .8)\right),\left(y_{4},(.9, .7)\right)\right\}\right\rangle, \\
\left\langle\bar{b}_{3},\left\{\left(y_{1},(.9, .8)\right),\left(y_{2},(.8, .9)\right),\left(y_{3},(.7, .8)\right),\left(y_{4},(.8, .9)\right)\right\}\right\rangle, \\
\left.\bar{b}_{4},\left\{\left(y_{1},(.7, .9)\right),\left(y_{2},(.8, .9)\right),\left(y_{3},(.9, .8)\right),\left(y_{4},(.9, .7)\right)\right\}\right\rangle,
\end{array}\right\}
\end{array}
$$

Tabular forms of q-ROF Hypersoft values are as follows:

\section{Table 1}

$\begin{array}{lllll}(\Re, \bar{A}) & y_{1} & y_{2} & y_{3} & y_{4} \\ \left(\mathrm{a}_{11}, \mathrm{a}_{21}, \mathrm{a}_{31}\right) & (.6, .8) & (.7, .9) & (.8, .6) & (.5, .9) \\ \left(\mathrm{a}_{11}, \mathrm{a}_{21}, \mathrm{a}_{32}\right) & (.5, .8) & (.7, .6) & (.9, .5) & (.9, .8) \\ \left(\mathrm{a}_{12}, \mathrm{a}_{21}, \mathrm{a}_{31}\right) & (.6, .8) & (.7, .6) & (.7, .9) & (.6, .9) \\ \left(\mathrm{a}_{12}, \mathrm{a}_{21}, \mathrm{a}_{32}\right) & (.9, .7) & (.9, .7) & (.7, .8) & (.8, .9)\end{array}$

\begin{tabular}{|c|c|c|c|c|}
\hline $\bar{\beta})$ & & & & \\
\hline & $, .7)$ & $, .9)$ & $7, .9)$ & \\
\hline & $(.7, .8)$ & $(.7, .8)$ & $(.9, .8)$ & $9, .7$ \\
\hline & $(.9, .8)$ & $(.8, .9)$ & $(.7, .8)$ & $8, .9$ \\
\hline & $(.7, .9)$ & $(.8, .9)$ & $(.9, .8)$ & $(.9, .7$ \\
\hline
\end{tabular}

Table 2

Definition 3.2. A q-ROF Hypersoft sets $(\Re, \bar{A})$ over $Y$ is said to be null q-ROF Hypersoft set and denoted by $0\left(Y_{q-R O F H S}, \bar{A}\right)$ if for all $\ddot{y} \in Y$ and $\bar{a} \in \bar{A}, \psi_{\bar{A}(\bar{a})}(\ddot{y})=0$ and $\tilde{\psi}_{\bar{A}(\bar{a})}(\ddot{y})=1$.

Definition 3.3. A q-ROF Hypersoft sets $(\Re, \bar{A})$ over $Y$ is said to be absolute q-ROF Hypersoft sets and denoted by $1\left(Y_{q-R O F H S}, \bar{A}\right)$ if for all $\ddot{y} \in Y$ and $\bar{a} \in \bar{A}, \psi_{\bar{A}(\bar{a})}(\ddot{y})=1$ and $\tilde{\psi}_{\bar{A}(\bar{a})}(\ddot{y})$.

Corollary 3.4. It is clear that each $q-R O F$ Hypersoft set is also $q-R O F$ soft set. An example of this situation is pointed below.

Example 2 We consider that Example 1. Suppose we select the parameters from a single attribute set $\tau_{1}$ while creating the q-rung orthopair fuzzy hypersoft set. In that case, the resulting set becomes the q-rung orthopair fuzzy soft set. Therefore, it is clear that each q-rung orthopair fuzzy hypersoft set is also a q-rung orthopair fuzzy soft set. The q-rung orthopair fuzzy hypersoft set structure is the generalized version of the q-rung orthopair fuzzy soft set. 
Definition 3.5. Two q-ROF Hypersoft set $\left(\Re, \bar{A}_{1}\right)$ and $\left(G, \bar{A}_{2}\right)$ over the universe $Y$ is said to be equal $q$-ROF Hypersoft sets and denoted by $\left(\Re, \bar{A}_{1}\right) \cong\left(G, \bar{A}_{2}\right)$ if for all $\ddot{y} \in Y$ and $\bar{a} \in \bar{A}, \psi_{\bar{A}_{1}(\bar{a})}(\ddot{y})=\psi_{\bar{A}_{2}(\bar{a})}(\ddot{y})$ and $\tilde{\psi}_{\left(\Re, \bar{A}_{1}\right)}(\ddot{y})=\tilde{\psi}_{\left(G, \bar{A}_{2}\right)}(\ddot{y})$.

Theorem 3.6. Let $Y$ be a universe set and $\left(\Re, \bar{A}_{1}\right),\left(G, \bar{A}_{2}\right),\left(K, \bar{A}_{3}\right)$ be three $q$-ROF Hypersoft sets over the universe $Y$. Then,

i) $\left(\Re, \bar{A}_{1}\right) \subseteq \underline{\subseteq} 1_{\left(Y_{q-R O F H S}, \bar{A}\right)}$,

ii) $0_{\left(Y_{q-R O F H S}, \bar{A}\right)} \tilde{\subseteq}\left(\Re, \bar{A}_{1}\right)$

iii) $\left(\Re, \bar{A}_{1}\right) \tilde{\subseteq}\left(G, \bar{A}_{2}\right)$ and $\left(G, \bar{A}_{2}\right) \tilde{\subseteq}\left(K, \bar{A}_{3}\right) \Rightarrow\left(\Re, \bar{A}_{1}\right) \tilde{\subseteq}\left(K, \bar{A}_{3}\right)$.

Proof i) $\left(\Re, \bar{A}_{1}\right) \subseteq \tilde{\subseteq}_{\left(Y_{q-R O F H S}, \bar{A}\right)}$, since $\psi_{\bar{A}_{1}(\bar{a})}(\ddot{y}) \leq \psi_{\bar{A}(\bar{a})}(\ddot{y})=1$ and $\tilde{\psi}_{\bar{A}_{1}(\bar{a})}(\ddot{y}) \geq$ $\tilde{\psi}_{\bar{A}(\bar{a})}(\ddot{y})=0$, for all $\overline{\mathrm{a}} \in \bar{A}, \ddot{y} \in Y$.

ii) $0_{\left(Y_{q-R O F H S}, \bar{A}\right)} \tilde{\subseteq}\left(\Re, \bar{A}_{1}\right)$, since $0=\psi_{\bar{A}_{1}(\bar{a})}(\ddot{y}) \leq \psi_{\bar{A}(\bar{a})}(\ddot{y})$ and $1=\tilde{\psi}_{\bar{A}_{1}(\bar{a})}(\ddot{y}) \geq \tilde{\psi}_{\bar{A}(\bar{a})}(\ddot{y})$ for all $\bar{a} \in \bar{A}, \ddot{y} \in Y$.

iii) $\left(\Re, \bar{A}_{1}\right) \subseteq \tilde{\subseteq}\left(G, \bar{A}_{2}\right) \Rightarrow \psi_{\bar{A}_{1}(\bar{a})}(\ddot{y}) \leq \psi_{\bar{A}_{2}(\bar{a})}(\ddot{y})$ and $\tilde{\psi}_{\bar{A}_{2}(\bar{a})}(\ddot{y}) \geq \tilde{\psi}_{\bar{A}_{1}(\bar{a})}(\ddot{y})$ for all $\bar{a} \in \bar{A}, \ddot{y} \in Y .\left(G, \bar{A}_{2}\right) \tilde{\subseteq}\left(K, \bar{A}_{3}\right) \Rightarrow \psi_{\bar{A}_{2}(\bar{a})}(\ddot{y}) \leq \psi_{\bar{A}_{3}(\bar{a})}(\ddot{y})$ and $\tilde{\psi}_{\bar{A}_{3}(\bar{a})}(\ddot{y}) \geq \tilde{\psi}_{\bar{A}_{2(\bar{a})}}(\ddot{y})$ for all $\bar{a} \in \bar{A}, \ddot{y} \in Y$. Therefore $\psi_{\bar{A}_{2}(\bar{a})}(\ddot{y}) \leq \psi_{\bar{A}_{3}(\bar{a})}(\ddot{y})$ and $\tilde{\psi}_{\bar{A}_{1}(\bar{a})}(\ddot{y}) \geq \tilde{\psi}_{\bar{A}_{3}(\bar{a})}(\ddot{y})$.Thus, we obtain $\left(\Re, \bar{A}_{1}\right) \tilde{\subseteq}\left(K, \bar{A}_{3}\right)$.

Definition 3.7. Let $Y$ be a universe of discourse and $\left(\Re_{1}, \bar{A}\right),\left(\Re_{2}, \bar{\beta}\right)$ be two q-ROF Hypersoft sets over $Y$. Then, $\left(\Re_{1}, \bar{A}\right)$ is a q-ROF Hypersoft subset of $\left(\Re_{2}, \bar{\beta}\right)$, which are define as $\left(\Re_{1}, \bar{A}\right) \tilde{\subseteq}\left(\Re_{2}, \bar{\beta}\right):$ if

- $\bar{A} \subseteq \bar{B}$

- for any $\bar{a} \in \bar{A}, \Re_{1}(\bar{a}) \subseteq \Re_{2}(\bar{a})$

Example 3 We consider that attributes in Example 1 and let $(\Re, \bar{A})$ and $(G, \bar{\beta})$ be two q-Rung orthopair Fuzzy hypersoft sets over $Y=\left\{y_{1}, y_{2}, y_{3}, y_{4}\right\}$. Tabular forms of $(\Re, \bar{A})$ and $(G, \bar{\beta})$ are following:

\section{Table 3}

$$
\begin{array}{lllll}
(\Re, \bar{A}) & y_{1} & y_{2} & y_{3} & y_{4} \\
\left(\mathrm{a}_{11}, \mathrm{a}_{21}, \mathrm{a}_{31}\right) & (.8, .9) & (.7, .9) & (.7, .8) & (.8, .9) \\
\left(\mathrm{a}_{11}, \mathrm{a}_{21}, \mathrm{a}_{32}\right) & (.7, .8) & (.6, .9) & (.7, .9) & (.7, .8) \\
\left(\mathrm{s}_{12}, \mathrm{a}_{21}, \mathrm{a}_{31}\right) & (.7, .9) & (.8, .9) & (.7, .8) & (.7, .9) \\
\left(\mathrm{a}_{12}, \mathrm{a}_{21}, \mathrm{a}_{32}\right) & (.8, .9) & (.7, .9) & (.7, .9) & (.8, .9)
\end{array}
$$

Table 4

$$
\begin{array}{lllll}
(G, \bar{\beta}) & y_{1} & y_{2} & y_{3} & y_{4} \\
\left(\mathrm{a}_{11}, \mathrm{a}_{21}, \mathrm{a}_{31}\right) & (.9, .8) & (.9, .8) & (.8, .7) & (.9, .7) \\
\left(\mathrm{a}_{11}, \mathrm{a}_{21}, \mathrm{a}_{32}\right) & (.8, .7) & (.8, .7) & (.8, .7) & (.8, .7) \\
\left(\mathrm{a}_{11}, \mathrm{a}_{22}, \mathrm{a}_{31}\right) & (.8, .8) & (.9, .7) & (.9, .7) & (.8, .7) \\
\left(\mathrm{a}_{11}, \mathrm{a}_{22}, \mathrm{a}_{32}\right) & (.9, .8) & (.9, .8) & (.9, .8) & (.9, .8)
\end{array}
$$

It is clear that $(\Re, \bar{A}) \subseteq(G, \bar{\beta})$. 
Definition 3.8. Let $Y$ be a set of discourse and $(\Re, \bar{A})$ and $(G, \bar{\beta})$ be two q-ROF Hypersoft sets over $Y$. The union of $(\Re, \bar{A})$ and $(G, \bar{\beta})$ is defined as: $(\Re, \bar{A}) \tilde{\cup}(G, \bar{\beta})=(K, \bar{C})$ and $\bar{C}=\bar{A} \cup \bar{\beta}$

$$
\begin{gathered}
\psi_{K(\bar{a})}(\ddot{y})=\left\{\begin{array}{c}
\Re(\bar{a}) \text { if } \bar{a} \in \bar{A}-\bar{\beta} \\
G(\bar{a}) \text { if } \bar{a} \in \bar{\beta}-\bar{A} \\
\max (\Re(\bar{a}), G(\bar{a})) \text { if } \bar{a} \in \bar{A} \cap \bar{\beta}
\end{array}\right. \\
\tilde{\psi}_{K(\bar{a})}(\ddot{y})=\left\{\begin{array}{c}
\Re(\bar{a}) \text { if } \bar{a} \in \bar{A}-\bar{\beta} \\
G(\bar{a}) \text { if } \bar{a} \in \bar{\beta}-\bar{A} \\
\min (\Re(\bar{a}), G(\bar{a})) \text { if } \bar{a} \in \bar{A} \cap \bar{\beta}
\end{array}\right.
\end{gathered}
$$

Example 4 We consider that attributes in Example 1 over the universe $Y=\left\{y_{1}, y_{2}, y_{3}, y_{4}\right\}$ and let $(\Re, \bar{A})$ and $(G, \bar{B})$ be two q-ROF hypersoft set, which are defined as follows:

$$
\begin{array}{r}
(\Re, \bar{A})=\left\{\begin{array}{l}
\left\langle\bar{a}_{1},\left\{\left(y_{1},(.8, .7)\right),\left(y_{2},(.8, .7)\right),\left(y_{3},(.8, .7)\right),\left(y_{4},(.9, .7)\right)\right\}\right\rangle, \\
\left\langle\bar{a}_{2},\left\{\left(y_{1},(.6, .8)\right),\left(y_{2},(.7, .8)\right),\left(y_{3},(.9, .8)\right),\left(y_{4},(.9, .6)\right)\right\}\right\rangle, \\
\left\langle\bar{a}_{3},\left\{\left(y_{1},(.9, .7)\right),\left(y_{2},(.6, .8)\right),\left(y_{3},(.7, .9)\right),\left(y_{4},(.8, .9)\right)\right\}\right\rangle, \\
\left\langle\bar{a}_{4},\left\{\left(y_{1},(.7, .9)\right),\left(y_{2},(.8, .7)\right),\left(y_{3},(.9, .8)\right),\left(y_{4},(.8, .7)\right)\right\}\right\rangle,
\end{array}\right\} \\
(G, \overline{\boldsymbol{B}})=\left\{\begin{array}{l}
\left\langle\bar{b}_{1},\left\{\left(y_{1},(.9, .7)\right),\left(y_{2},(.8, .9)\right),\left(y_{3},(.7, .9)\right),\left(y_{4},(.8, .7)\right)\right\}\right\rangle, \\
\left\langle\bar{b}_{2},\left\{\left(y_{1},(.7, .8)\right),\left(y_{2},(.7, .8)\right),\left(y_{3},(.9, .8)\right),\left(y_{4},(.9, .7)\right)\right\}\right\rangle, \\
\left\langle\bar{b}_{3},\left\{\left(y_{1},(.9, .8)\right),\left(y_{2},(.8, .9)\right),\left(y_{3},(.7, .8)\right),\left(y_{4},(.8, .9)\right)\right\}\right\rangle, \\
\left\langle\bar{b}_{4},\left\{\left(y_{1},(.7, .9)\right),\left(y_{2},(.8, .9)\right),\left(y_{3},(.9, .8)\right),\left(y_{4},(.9, .7)\right)\right\}\right\rangle,
\end{array}\right\}
\end{array}
$$

The union of two q-ROF hypersoft sets is written as:

$$
(\Re, \bar{A}) \cup(G, \bar{B})=\left\{\begin{aligned}
& \left\langle\bar{c}_{1},\left\{\left(y_{1},(.9, .7)\right),\left(y_{2},(.8, .7)\right),\left(y_{3},(.8, .7)\right),\left(y_{4},(.9, .7)\right)\right\}\right\rangle, \\
& \left\langle\bar{c}_{2},\left\{\left(y_{1},(.7, .8)\right),\left(y_{2},(.7, .8)\right),\left(y_{3},(.9, .8)\right),\left(y_{4},(.9, .6)\right)\right\}\right\rangle, \\
& \left\langle\bar{c}_{3},\left\{\left(y_{1},(.9, .7)\right),\left(y_{2},(.6, .8)\right),\left(y_{3},(.7, .9)\right),\left(y_{4},(.8, .9)\right)\right\}\right\rangle, \\
& \left\langle\bar{c}_{4},\left\{\left(y_{1},(.7, .9)\right),\left(y_{2},(.8, .7)\right),\left(y_{3},(.9, .8)\right),\left(y_{4},(.8, .7)\right)\right\}\right\rangle, \\
& \left\langle\bar{c}_{5},\left\{\left(y_{1},(.9, .8)\right),\left(y_{2},(.8, .9)\right),\left(y_{3},(.7, .8)\right),\left(y_{4},(.8, .9)\right)\right\}\right\rangle, \\
& \left\langle\bar{c}_{6},\left\{\left(y_{1},(.7, .9)\right),\left(y_{2},(.8, .9)\right),\left(y_{3},(.9, .8)\right),\left(y_{4},(.9, .7)\right)\right\}\right\rangle,
\end{aligned}\right\}
$$

Definition 3.9. Let $Y$ be a set of discourse and $(\Re, \bar{A})$ and $(G, \bar{\beta})$ be two q-ROF Hypersoft sets over $Y$. The intersection of $(\Re, \bar{A})$ and $(G, \bar{\beta})$ is defined as: $(\Re, \bar{A}) \tilde{\cap}(G, \bar{\beta})=(K, \bar{C})$ and $\bar{C}=\bar{A} \cap \bar{\beta}$

$(K, \bar{C})=\left\{\left\langle\ddot{y},\left(\min \left\{\psi_{\bar{A}(\bar{a})}(\ddot{y}), \psi_{\bar{\beta}(\bar{b})}(\ddot{y})\right\}, \max \left\{\tilde{\psi}_{\bar{A}(\bar{a})}(\ddot{y}), \tilde{\psi}_{\bar{\beta}(\bar{b})}(\ddot{y})\right\}\right)\right\rangle \mid \ddot{y} \in Y, \bar{a} \in \bar{A}\right.$ and $\left.\bar{b} \in \bar{\beta}\right\}$

Example 5 We consider that attributes in Example 1 over the universe $Y=\left\{y_{1}, y_{2}, y_{3}, y_{4}\right\}$ and let $(\Re, \bar{A})$ and $(G, \bar{\beta})$ be two q-ROF hypersoft sets, which are defined as follows:

$$
\begin{array}{r}
(\Re, \bar{A})=\left\{\begin{array}{l}
\left\langle\bar{a}_{1},\left\{\left(y_{1},(.8, .7)\right),\left(y_{2},(.8, .7)\right),\left(y_{3},(.8, .7)\right),\left(y_{4},(.9, .7)\right)\right\}\right\rangle, \\
\left\langle\bar{a}_{2},\left\{\left(y_{1},(.6, .8)\right),\left(y_{2},(.7, .8)\right),\left(y_{3},(.9, .8)\right),\left(y_{4},(.9, .6)\right)\right\}\right\rangle, \\
\left\langle\bar{a}_{3},\left\{\left(y_{1},(.9, .7)\right),\left(y_{2},(.6, .8)\right),\left(y_{3},(.7, .9)\right),\left(y_{4},(.8, .9)\right)\right\}\right\rangle, \\
\left\langle\bar{a}_{4},\left\{\left(y_{1},(.7, .9)\right),\left(y_{2},(.8, .7)\right),\left(y_{3},(.9, .8)\right),\left(y_{4},(.8, .7)\right)\right\}\right\rangle,
\end{array}\right\} \\
(G, \bar{\beta})=\left\{\begin{array}{l}
\left\langle\bar{b}_{1},\left\{\left(y_{1},(.9, .7)\right),\left(y_{2},(.8, .9)\right),\left(y_{3},(.7, .9)\right),\left(y_{4},(.8, .7)\right)\right\}\right\rangle, \\
\left\langle\bar{b}_{2},\left\{\left(y_{1},(.7, .8)\right),\left(y_{2},(.7, .8)\right),\left(y_{3},(.9, .8)\right),\left(y_{4},(.9, .7)\right)\right\}\right\rangle, \\
\left\langle\bar{b}_{3},\left\{\left(y_{1},(.9, .8)\right),\left(y_{2},(.8, .9)\right),\left(y_{3},(.7, .8)\right),\left(y_{4},(.8, .9)\right)\right\}\right\rangle, \\
\left\langle\bar{b}_{4},\left\{\left(y_{1},(.7, .9)\right),\left(y_{2},(.8, .9)\right),\left(y_{3},(.9, .8)\right),\left(y_{4},(.9, .7)\right)\right\}\right\rangle,
\end{array}\right\}
\end{array}
$$


The intersection of two q-ROF hypersoft sets is written as:

$$
(\Re, \bar{A}) \cap(G, \bar{\beta})=\left\{\begin{array}{l}
\left\langle\bar{c}_{1},\left\{\left(y_{1},(.8, .7)\right),\left(y_{2},(.8, .9)\right),\left(y_{3},(.7, .9)\right),\left(y_{4},(.8, .7)\right)\right\}\right\rangle, \\
\left\langle\bar{c}_{2},\left\{\left(y_{1},(.6, .8)\right),\left(y_{2},(.7, .8)\right),\left(y_{3},(.9, .8)\right),\left(y_{4},(.9, .7)\right)\right\}\right\rangle,
\end{array}\right\}
$$

Definition 3.10. Let $(\Re, \bar{A})$ and $(G, \bar{\beta})$ be two q-ROF Hypersoft sets over Y. The "OR" operation on them is denoted by

$$
(\Re, \bar{A}) \vee(G, \bar{\beta})=(K, \bar{A} \times \bar{\beta})=(K, \bar{a} \times \bar{b})
$$

where

$$
\begin{aligned}
(K, \bar{a} \times \bar{b}) & =\Re_{\bar{A}(\bar{a})} \cup G_{\bar{\beta}(\bar{b})} \forall(\bar{a} \times \bar{b}) \in \bar{A} \times \bar{\beta} \\
& =\left\{\left\{\max \left(\psi_{\Re(\bar{a})}, \psi_{G(\bar{b})}\right)\right\},\left\{\min \left(\tilde{\psi}_{\Re(\bar{a})}, \tilde{\psi}_{G(\bar{b})}\right)\right\} \mid \ddot{y} \in Y, \text { and } \bar{a}, \bar{b} \in \bar{A}, \bar{\beta}\right\}
\end{aligned}
$$

Example 6 We consider that attributes in Example 1 over the universe $Y=\left\{y_{1}, y_{2}\right\}$ and let $(\Re, \bar{A})$ and $(G, \bar{B})$ be two q-ROF hypersoft set, which are defined as follows:

\begin{tabular}{|c|c|c|}
\hline$(\Re, \bar{A})$ & & $y_{2}$ \\
\hline $\bar{a}_{1}$ & $(.8, .7)$ & $(.8, .7)$ \\
\hline $\bar{a}_{2}$ & $(.6, .8)$ & $(.7, .8)$ \\
\hline $\bar{a}_{3}$ & $(.8, .7)$ & $(.6, .9)$ \\
\hline
\end{tabular}

$$
\begin{array}{r}
(\Re, \bar{A})=\left\{\begin{array}{l}
\left\langle\bar{a}_{1},\left\{\left(y_{1},(.8, .7)\right),\left(y_{2},(.8, .7)\right)\right\}\right\rangle, \\
\left\langle\bar{a}_{2},\left\{\left(y_{1},(.6, .8)\right),\left(y_{2},(.7, .8)\right)\right\}\right\rangle, \\
\left\langle\bar{a}_{3},\left\{\left(y_{1},(.8, .7)\right),\left(y_{2},(.6, .9)\right)\right\}\right\rangle,
\end{array}\right\} \\
(G, \overline{\boldsymbol{\beta}})=\left\{\begin{array}{l}
\left\langle\bar{b}_{1},\left\{\left(y_{1},(.9, .7)\right),\left(y_{2},(.8, .9)\right)\right\}\right\rangle, \\
\left\langle\bar{b}_{2},\left\{\left(y_{1},(.7, .8)\right),\left(y_{2},(.7, .8)\right)\right\}\right\rangle, \\
\left\langle\bar{b}_{3},\left\{\left(y_{1},(.9, .8)\right),\left(y_{2},(.8, .9)\right)\right\}\right\rangle,
\end{array}\right\}
\end{array}
$$

Tabular forms q-ROF hypersoft sets are as follows:

\section{Table 5}

\section{Table 6}

$\begin{array}{lll}(\mathrm{G}, \bar{\beta}) & y_{1} & y_{2} \\ \bar{b}_{1} & (.9, .7) & (.8, .9) \\ \bar{b}_{2} & (.7, .8) & (.7, .8) \\ \bar{b}_{3} & (.9, .8) & (.8, .9)\end{array}$


The "OR" operation of q-ROF Hypersoft sets is given as follows:

Table 7

$\begin{array}{lll}(\Re, \bar{A}) \vee(G, \bar{\beta}) & y_{1} & y_{2} \\ \bar{a}_{1} \times \bar{b}_{1} & (.9, .7) & (.8, .7) \\ \bar{a}_{1} \times \bar{b}_{2} & (.8, .7) & (.8, .7) \\ \bar{a}_{1} \times \bar{b}_{3} & (.9, .7) & (.8, .7) \\ \bar{a}_{2} \times \bar{b}_{1} & (.9, .7) & (.8, .8) \\ \bar{a}_{2} \times \bar{b}_{2} & (.7, .8) & (.7, .8) \\ \bar{a}_{2} \times \bar{b}_{3} & (.9, .8) & (.8, .8) \\ \bar{a}_{3} \times \bar{b}_{1} & (.9, .7) & (.8, .9) \\ \bar{a}_{3} \times \bar{b}_{2} & (.8, .7) & (.7, .8) \\ \bar{a}_{3} \times \bar{b}_{3} & (.9, .7) & (.8, .9)\end{array}$

Definition 3.11. Let $(\Re, \bar{A})$ and $(G, \bar{\beta})$ be two q-ROF Hypersoft sets over $Y$. The "AND” operation on them is denoted by

$$
(\Re, \bar{A}) \wedge(G, \bar{\beta})=(K, \bar{A} \times \bar{\beta})=(K, \bar{a} \times \bar{b})
$$

where

$$
\begin{aligned}
(K, \bar{a} \times \bar{b}) & =\Re_{\bar{A}(\bar{a})} \cup G_{\bar{\beta}(\bar{b})} \forall(\bar{a} \times \bar{b}) \in \bar{A} \times \bar{\beta} \\
& =\left\{\left\{\min \left(\psi_{\Re(\bar{a})}, \psi_{G(\bar{b})}\right)\right\},\left\{\max \left(\tilde{\psi}_{\Re(\bar{a})}, \tilde{\psi}_{G(\bar{b})}\right)\right\} \mid \ddot{y} \in Y \text {, and } \bar{a}, \bar{b} \in \bar{A}, \bar{\beta}\right\}
\end{aligned}
$$

Example 7 We consider that attributes in Example 1 over the universe $Y=\left\{y_{1}, y_{2}\right\}$ and let $(\Re, \bar{A})$ and $(G, \bar{B})$ be two q-ROF hypersoft sets, which are defined as follows:

$$
\begin{array}{r}
(\Re, \bar{A})=\left\{\begin{array}{l}
\left\langle\bar{a}_{1},\left\{\left(y_{1},(.8, .7)\right),\left(y_{2},(.8, .7)\right)\right\}\right\rangle, \\
\left\langle\bar{a}_{2},\left\{\left(y_{1},(.6, .8)\right),\left(y_{2},(.7, .8)\right)\right\}\right\rangle, \\
\left\langle\bar{a}_{3},\left\{\left(y_{1},(.8, .7)\right),\left(y_{2},(.6, .9)\right)\right\}\right\rangle,
\end{array}\right\} \\
(G, \bar{\beta})=\left\{\begin{array}{l}
\left\langle\bar{b}_{1},\left\{\left(y_{1},(.9, .7)\right),\left(y_{2},(.8, .9)\right)\right\}\right\rangle, \\
\left\langle\bar{b}_{2},\left\{\left(y_{1},(.7, .8)\right),\left(y_{2},(.7, .8)\right)\right\}\right\rangle, \\
\left\langle\bar{b}_{3},\left\{\left(y_{1},(.9, .8)\right),\left(y_{2},(.8, .9)\right)\right\}\right\rangle,
\end{array}\right\}
\end{array}
$$

\begin{tabular}{|c|c|c|}
\hline$(\Re, \bar{A})$ & $y_{1}$ & $y_{2}$ \\
\hline $\bar{a}_{1}$ & $(.8, .7)$ & $(.8, .7)$ \\
\hline $\bar{a}_{2}$ & $(.6, .8)$ & $(.7, .8)$ \\
\hline $\bar{a}_{3}$ & $(.8, .7)$ & $(.6, .9)$ \\
\hline
\end{tabular}

Tabular forms q-ROF hypersoft sets are as follows:

\section{Table 8}

Table 9

$\begin{array}{lll}(\mathrm{G}, \overline{\boldsymbol{B}}) & y_{1} & y_{2} \\ \bar{b}_{1} & (.9, .7) & (.8, .9) \\ \bar{b}_{2} & (.7, .8) & (.7, .8) \\ \bar{b}_{3} & (.9, .8) & (.8, .9)\end{array}$


The "AND" operator of q-ROF Hypersoft sets is given as follows:

Table 10

$\begin{array}{lll}(\Re, \bar{A}) \wedge(G, \bar{\beta}) & y_{1} & y_{2} \\ \bar{a}_{1} \times \bar{b}_{1} & (.8, .7) & (.8, .9) \\ \bar{a}_{1} \times \bar{b}_{2} & (.7, .8) & (.7, .8) \\ \bar{a}_{1} \times \bar{b}_{3} & (.8, .8) & (.8, .9) \\ \bar{a}_{2} \times \bar{b}_{1} & (.6, .8) & (.7, .9) \\ \bar{a}_{2} \times \bar{b}_{2} & (.6, .8) & (.7, .8) \\ \bar{a}_{2} \times \bar{b}_{3} & (.6, .8) & (.7, .9) \\ \bar{a}_{3} \times \bar{b}_{1} & (.8, .7) & (.6, .9) \\ \bar{a}_{3} \times \bar{b}_{2} & (.7, .8) & (.6, .9) \\ \bar{a}_{3} \times \bar{b}_{3} & (.8, .8) & (.6, .9)\end{array}$

Definition 3.12. Let $(\Re, \bar{A})$ be q-ROF Hypersoft sets over $Y$. Then Complement of $(\Re, \bar{A})$ denoted by $(\Re, \bar{A})^{C}$ and is defined as $\left(\Re^{C}, \bar{A}\right)$ and its mapping is defined as

$$
\Re^{C}: \bar{A} \rightarrow q-R O F S^{Y}
$$

where ROF $S^{Y}$ represents the family of all subsets of $q$-ROF Hypersoft sets of $Y$. Where complement of $(\Re, \bar{A})=\left\{\ddot{y},\left(\psi_{\bar{A}(\bar{a})}, \tilde{\psi}_{\bar{A}(\bar{a})}\right) \mid \ddot{y} \in Y, \bar{a} \in \bar{A}\right\}$ is defined as $(\Re, \bar{A})^{C}=\left\{\ddot{y},\left(\tilde{\psi}_{\bar{A}(\bar{a})}, \psi_{\bar{A}(\bar{a})}\right) \mid \ddot{y} \in Y, \bar{a} \in \bar{A}\right\}$

Example 8 Consider the q-ROF hypersoft set $(\Re, \bar{A})$ in Example 1 over the universe $Y=\left\{y_{1}, y_{2}, y_{3}, y_{4}\right\}$.

$$
(\Re, \bar{A})=\left\{\begin{array}{l}
\left\langle\bar{a}_{1},\left\{\left(y_{1},(.8, .7)\right),\left(y_{2},(.8, .7)\right),\left(y_{3},(.8, .7)\right),\left(y_{4},(.9, .7)\right)\right\}\right\rangle, \\
\left\langle\bar{a}_{2},\left\{\left(y_{1},(.6, .8)\right),\left(y_{2},(.7, .8)\right),\left(y_{3},(.9, .8)\right),\left(y_{4},(.9, .6)\right)\right\}\right\rangle, \\
\left\langle\bar{a}_{3},\left\{\left(y_{1},(.9, .7)\right),\left(y_{2},(.6, .8)\right),\left(y_{3},(.7, .9)\right),\left(y_{4},(.8, .9)\right)\right\}\right\rangle, \\
\left\langle\bar{a}_{4},\left\{\left(y_{1},(.7, .9)\right),\left(y_{2},(.8, .7)\right),\left(y_{3},(.9, .8)\right),\left(y_{4},(.8, .7)\right)\right\}\right\rangle,
\end{array}\right\}
$$

Then the complement of $(\Re, \bar{A})$ can be written as:

$$
(\Re, \bar{A})^{C}=\left\{\begin{array}{l}
\left\langle\bar{a}_{1},\left\{\left(y_{1},(.7, .8)\right),\left(y_{2},(.7, .8)\right),\left(y_{3},(.7, .8)\right),\left(y_{4},(.7, .9)\right)\right\}\right\rangle, \\
\left\langle\bar{a}_{2},\left\{\left(y_{1},(.8, .6)\right),\left(y_{2},(.8, .7)\right),\left(y_{3},(.8, .9)\right),\left(y_{4},(.6, .9)\right)\right\}\right\rangle, \\
\left\langle\bar{a}_{3},\left\{\left(y_{1},(.7, .9)\right),\left(y_{2},(.8, .6)\right),\left(y_{3},(.9, .7)\right),\left(y_{4},(.9, .8)\right)\right\}\right\rangle, \\
\left\langle\bar{a}_{4},\left\{\left(y_{1},(.9, .7)\right),\left(y_{2},(.7, .8)\right),\left(y_{3},(.8, .9)\right),\left(y_{4},(.7, .8)\right)\right\}\right\rangle,
\end{array}\right\}
$$

Definition 3.13. Let $(\Re, \bar{A})$ be $q$-ROF Hypersoft set over $Y$. Then Relative Complement of $(\Re, \bar{A})$ denoted by $\left(\Re^{r}, \bar{A}\right)$, such that $\Re^{r}: \bar{A} \rightarrow q-R O F S^{Y}$ is a mapping, shown by $\Re^{r}(\bar{a})=Y-\Re(\bar{a})$, for all $\bar{a} \in \bar{A}$, where $q-R O F S^{Y}$ represents the family of all subsets of q-ROF Hypersoft sets of $Y$. A pair $\left(\Re^{r}, \bar{A}\right)$ can be written as

$$
\left(\Re^{r}, \bar{A}\right)=\left\{\left\langle\bar{a}, \Re^{r}(\bar{a})\right\rangle \mid \bar{a} \in \bar{A}, \Re^{r}(\bar{a}) \in q-R O F H S^{Y}\right\}
$$

Theorem 3.14. i) Let $\left(\Re^{c}, \bar{A}\right)^{c}=(\Re, \bar{A})$

ii) $0_{(Y, \bar{A})}^{c}=1_{(Y, \bar{A})}$

iii) $1_{(Y, \bar{A})}^{c}=0_{(Y, \bar{A})}$

Proof Proofs are trivial. 


\section{RIGOROUS OF THE STUDY}

In real-world applications, the attributes must be sub-divided into attribute values for explicit knowledge. As a generalization of a soft set, the hypersoft set overcomes this limitation and emphasizes disjoint attribute value sets for various characteristics. This generalization demonstrates that the hypersoft set associated with q-rung orthopair fuzzy set theory will be extremely useful in building a link between alternatives and attributes. It ís interesting to note that the hypersoft approach can be used to any decision-making problem, regardless of the decision-maker's ability to select values. Multi-criteria decision making, Multi-criteria group decision making, medical diagnostics, probability theory, employee selection, and many more applications benefit from this the

\section{LiMITATIONS OF THE STUDY}

Although this structure can handle decision-making more effectively than fuzzy sets and soft sets, it isn't straightforward to use it manually. It is also time-consuming when taking it manually. So, to avoid such limitations, we need to develop some computer programming.

\section{CONCLUSION}

The purpose of this article is to overcome the uncertainty problem in a more particular way by combining q-ROF sets with hypersoft sets. q-ROF Hypersoft sets can accurately represent expert evaluation information and reduce fuzziness on decision results. Some operations of q-ROF Hypersoft sets such as subset, equal set, union, intersection, complement AND, OR are defined and checked its feasibility and effectiveness by numerical examples. We presented the basic properties of the q-ROF Hypersoft sets. An appropriate instance validates the validity and implementation of the proposed operations and definitions. We hope that this study will play a vital role in future decision-making techniques.

\section{REFERENCES}

[1] M. I. Ali, Another view on q-rung orthopair fuzzy sets, International Journal of Intelligent Systems 33, (2018) 2139-2153.

[2] K. T. Atanassov, Intuitionistic Fuzzy Sets, Fuzzy Sets and Systems 20 (1986) 87-96.

[3] P. Bhattacharya and N. P. Mukherjee, Fuzzy relations and fuzzy groups. Information sciences 36, No. 3 (1985) 267-282.

[4] N. Cagman, S. Enginoglu and F. Citak, Fuzzy soft set theory and its applications. Iranian journal of fuzzy systems, 8, No. 3 (2011) 137-147.

[5] M. Demirci, Fuzzy functions and their fundamental properties. Fuzzy Sets and Systems 106, No.2 (1999) 239-246

[6] K. Gong, X. Zhi and Z. Xia, The bijective soft set with its operations, Computers Mathematics with Applications 60, No. 8 (2010) 2270-2278.

[7] P. Liu and P. Wang, Some q-rung orthopair fuzzy aggregation operators and their applications to multipleattribute decision making, International Journal of Intelligent Systems 33, (2018) 259-280.

[8] P. K. Maji, Neutrosophic soft set. Annals of Fuzzy Mathematics and Informatics 5, No. 1, (2013) 157-168.

[9] D. Molodtsov, Soft set theory rst results. Computers and Mathematics with Applications 37, No. 4-5 (1999) 19-31.

[10] T. Y. Öztürk and A. Yolcu, On Neutrosophic Hypersoft Topological Spaces, Theory and Application of Hypersoft Set, (2021) 215.

[11] A. R. Roy and P. K. Maji, A fuzzy soft set theoretic approach to decision making problems. Journal of computational and Applied Mathematics, 203, No. 2 (2007) 412-418. 
[12] M. Saeed, M. Hussain, A. A. Mughal, A Study of Soft Sets with Soft Members and Soft Elements: A New Approach, Punjab Uni.j.math. 52 No. 8 (2020).

[13] M. Saeed, M. Ahsan, M. K. Siddique and A. R. Ahmad, A Study of the Fundamentals of Hypersoft Set Theory, International Journal of Scientific and Engineering Research 11, No.1 (2020) 320-329.

[14] M. Saeed, A.U. Rahman, M. Ahsan and F. Smarandache, An Inclusive Study on Fundamentals of Hypersoft Set, In Theory and Application of Hypersoft Set, Pons Publishing House, Brussel (2021) 1-23.

[15] M. Saeed, M. Ahsan and A.U. Rahman, A novel approach to mappings on hypersoft classes with application, In Theory and Application of Hypersoft Set, Pons Publication House, Brussel (2021) 175-191.

[16] F. Smarandache, Extension of soft set to hypersoft set, and then to plithogenic hypersoft set, Neutrosophic Sets Syst 22, (2018) 168-170.

[17] R. R. Yager, Generalized orthopair fuzzy sets, IEEE Transactions on Fuzzy Systems 25, (2016) 1222-1230.

[18] R. R. Yager, Pythagorean fuzzy subsets. In joint IFSA world congress and NAFIPS annual meeting (IFSA/NAFIPS) (2013) 57-61).

[19] R. R. Yager, Aggregation operators and fuzzy systems modeling. Fuzzy sets and systems 67, No. 2 (1994) 129-145.

[20] R. R. Yager and D. P. Filev, Essentials of fuzzy modeling and control. New York 388 (1994) 22-23

[21] A. Yolcu, and T. Y. Öztürk Fuzzy Hypersoft Sets and It's Application to Decision-Making, Theory and Application of Hypersoft Set, (2021) 50.

[22] A. Yolcu, F. Smrandache and T. Y. Öztürk, Intuitionistic fuzzy hypersoft sets, Communications Faculty of Sciences University of Ankara Series A1 Mathematics and Statistics, 70, No. 1 (2021) 443-455.

[23] L. A. Zadeh, Fuzzy Sets. Information and Control 8 (1965) 338353.

[24] R. M. Zulqarnain, X. L. Xin, M. Saeed, A Development of Pythagorean fuzzy hypersoft set with basic operations and decision-making approach based on the correlation coefficient. Theory and Application of Hypersoft Set No. 6 (2021). 